To cite this article: Akpabio Junior NC, Okeke UC, Epum CF, Okise CJ, Familoni OO, Oyekunle BJ, Abidakun MM, Okolo ID. Impact of COVID-19 (SARS-CoV-2) on the Surgical Care of Patients and Surgical Training in Africa. European Journal of Medical and Educational Technologies 2021; 14(2): em2105. https://doi.org/10.30935/ejmets/10810

\title{
Impact of COVID-19 (SARS-CoV-2) on the Surgical Care of Patients and Surgical Training in Africa
}

\author{
Nseobong Charles Akpabio Junior ${ }^{1 *}$ (D), Ugochukwu Chigozie Okeke ${ }^{1}$ (D), Chinweoge Frances Epum ${ }^{1}$ (D), \\ Chukwuamaka Jennifer Okise ${ }^{1}$ (D), Oluwaloni Olaitan Familoni ${ }^{1}$ (D), Babajide Joseph Oyekunle ${ }^{1}$ (D), \\ Modupeoluwa Mercy Abidakun ${ }^{1}$ (D), Isioma Dianne Okolo ${ }^{2}$ \\ ${ }^{1}$ Department of Medicine and Surgery, College of Medicine, University of Ibadan, Oyo State, Nigeria \\ 2 Program for Global Surgery and Social Change, Harvard Medical School, USA \\ * Corresponding author: Nseobong Charles Akpabio, Jr. E-mail: nseobongjunior3@gmail.com ORCID: 0000-0003-3796-0331 \\ Received: 26 November 2020 Accepted: 1 January 2021
}

\begin{abstract}
The Coronavirus pandemic poses a significant threat to the healthcare sectors of some African countries due to poor healthcare organisation, financing, and reduced uptake of recent technological advancements. Surgical care of patients and surgical training of healthcare workers are considerably affected, due to the dearth of policies and strategic health plans, to ensure the provision of safe and affordable surgical care and continuity of training. The purpose of this study is to explore the impact of the COVID-19 pandemic on Surgery in Africa and to provide recommendations geared towards the current pandemic and for the future. This review involved a search of the electronic databases MEDLINE/PubMed and Google Scholar, and 31 papers from African countries which explored the impact of COVID-19 across different surgical specialities were screened. The cancellation rate of elective surgeries and benign conditions across some countries were seen to be as high as $74-81 \%$ with prioritisation of cancer patients and emergencies. The volume of emergency surgical cases presenting in some hospitals was reduced due to the associated lockdowns and fear of contracting the virus, while Telemedicine became increasingly adopted with newer platforms being used across some countries. The pandemic has exposed the inequities in health systems and further studies need to be done to evaluate its impact across more surgical specialities.
\end{abstract}

Keywords: global health, surgery, health systems, health technology, COVID-19

\section{INTRODUCTION}

In December 2019, health professionals from Hubei Province in Wuhan city, the most populous city in China, reported the first 27 cases of pneumonia with an unidentified aetiology [1]. On the $7^{\text {th }}$ of January 2020, these cases were diagnosed as COVID-19 (SARS-CoV-2) by the Chinese Centre for Disease
Control and Prevention [1]. The World Health Organization (WHO) subsequently declared the COVID-19 outbreak a public health emergency of international concern on the 30th of January, 2020 and a pandemic on the $11^{\text {th }}$ of March, 2020 [1]. 
The 2019 novel coronavirus was first diagnosed in Africa on the $14^{\text {th }}$ of February 2020, when the first case reported in Egypt. [2]. Subsequently, the Africa Centre for Disease and Control jointly working with the Nigeria Centre for Disease and Control, various national public health agencies, and the $W H O$, began reinforcing preparation efforts to handle the impact of the pandemic. African countries like Nigeria, Kenya, the Democratic Republic of the Congo and South Africa, were identified as high-risk priority zones for proactive surveillance, detection, and containment of the spread of COVID-19 due to their close links with China [2]. These efforts were paramount because outbreaks due to emerging and re-emerging diseases have negatively impacted the health systems of African countries over the years, as was particularly observed during the Ebola virus Epidemic [2].

Due to poor health care planning, financing, and reduced uptake of recent technological health care advancements, African surgical care and training are very vulnerable to social, economic and political disruptions caused by the pandemic [3]. As at 2015 , only $2 \%$ of 4,064 targets in national health strategic plans from 43 African countries covered surgical conditions, and 33 percent of national health policies in these African countries had no surgical targets [4]. In Sub-Saharan Africa, the overall disease burden associated with surgical conditions is estimated to be 38 disabilityadjusted life years lost per 1,000. This is significantly higher than in other world regions. Trauma $(15 / 1,000)$, obstetric complications $(6 / 1,000)$, malignancies $(3 / 1,000)$, perinatal conditions $(3 / 1,000)$, congenital anomalies $(3 / 1,000)$, cataracts and glaucoma $(2 / 1,000)$ being mainly contributory [5]. Many African countries have fewer than 5 hospital beds per 10, 000 of the population [6]. Sub Saharan Africa possesses a surgical density of 0.5 surgeons per 100,000 individuals which is less than $1 \%$ of the surgeon density of high-income Organizations for Economic Co-operation and Development Nations [7].

The COVID-19 pandemic is estimated to lead to a loss of several billion dollars in gross domestic product for the African subcontinent [8]. Many African countries are still unable to meet the obligations of the Abuja declaration which aims to allocate at least $15 \%$ of their annual budget to health care and are currently unable to make the investments needed to scale up surgical access by 2030 [9]. $55 \%$ of surgical care in lower and middle-income countries is estimated to be from external international charitable organizations, and spending on local capacity building has remained low. With the associated lockdowns in various African countries, levels of poverty are likely to increase, leaving individuals more vulnerable to catastrophic out-ofpocket expenditures [9].

Africa's poor health care indices demonstrate the need to proactively address the surgical needs of patients during the pandemic to avoid deepening already existing inequalities and consequent predisposition to future pandemics. This article aims to explore the impact of the COVID-19 pandemic on Surgery in Africa and to provide recommendations geared towards the current pandemic and for the future.

\section{CANCELLATION OF EMERGENCY AND ELECTIVE SURGERIES}

At the start of the pandemic, the World Bank estimated the 12 -week cancellation rate of surgeries to be $68.3-73.0 \%$. The least rate of cancellation (520, 459 cases) was estimated to occur in Sub-Saharan Africa, showing the low volume of surgical support in this region and reflecting the unmet need of patients in Africa. Europe and Central Asia were estimated to have the most cancellations with about $8,430,348$ procedures [10]. Although hospitals in regions with better access to resources have postponed elective surgeries indefinitely to save essential resources, including hospital beds and personal protective equipment, this strategy is not so easily replicable in a large number of African facilities [11]. In the United States of America, an estimated $91 \%$ of surgeries are elective, in Europe, $75 \%$ of surgeries are considered elective and while in African countries, only $43 \%$ of surgical procedures are considered elective [12].

A large number of surgeries conducted in African countries are emergencies not elective [13]. Oncologists working in West Africa, South Africa and Sudan for example, have been faced with the dilemma of finding a compromise between protecting themselves, their staff and their patients from COVID-19, and providing essential surgical care for their patients $[13,14]$. In Sudan, cancer centres established a contingency plan by deferring new referrals except for emergency cases. Also, elective surgery, non-urgent intravenous chemotherapy, and follow-up visits were suspended for 2 weeks at the onset of the pandemic while scheduled appointments for patients on radiotherapy were maintained and inpatients were allowed to have only one visitor per day [14]. It has been particularly difficult for Oncologists working in African countries to manage patients effectively considering the scarcity of workforce, resources, and the lack of a centralised COVID-19 guideline for managing patients [14]. In most countries in Africa, hospitalized patients with a stable condition awaiting scheduled surgeries have been discharged and truly, 
elective surgical procedures have been suspended $[11,15,16]$. However, decision-making, as it pertains to the pursuance of elective surgeries, has been fluid, and each hospital makes those decisions based on good clinical judgement and the level of available resources [11]. Gynaecological endoscopy surgeries as another example have been postponed, with the consideration that there is an increased risk of transmission of COVID-19 during laparoscopic and hysteroscopic procedures [17]. In a crosssectional survey conducted in Nigeria, elective surgeries were suspended in $46(92 \%)$ of 50 centres at the time of the survey [16].

Restricting surgery to only emergency cases may be disadvantageous to surgeons in Africa. Due to relatively lower salaries in government institutions, many providers rely on elective and private work for their financial wellbeing, thus postponing elective surgery may work against their financial incentives [11] Furthermore, there has also been a noticeable reduction in the volume of emergency surgeries compared with what surgical departments usually had pre-pandemic $[15,16]$. The reduction in presentation of emergencies could be as a result of the public "lockdown", resulting in fewer accidents; however, the fear of contracting the disease could also proffer an explanation to the decrease in the presentation of other emergencies, such as tumours and vascular diseases [15]. In some countries, the number of surgical departments has been cut down. For instance, in Morocco, the number of neurosurgical departments have been reduced and their attention directed specifically to emergencies and critically ill patients. In some instances, up to $50 \%$ of the neurosurgical staff were incorporated into COVID-19 response units [15].

A multi-country study conducted by the COVIDSurg Collaborative suggested that across all surgical specialities, the proportions of surgery projected to be cancelled for benign conditions were higher compared to cancer surgeries. In African countries, the cancellation rate of elective surgeries for benign conditions across surgical specialities range from $74.2-81 \%$, and for cancer surgeries $39.5-71.6 \%$. Orthopaedic procedures have been cancelled most frequently, with 90000 orthopaedic surgeries cancelled over 12 weeks [10].

\section{IMPACT ON SURGICAL PERSONNEL AND TRAINING}

An observational study conducted in Eastern Nigeria revealed that $88 \%$ of surgeons surveyed have not been involved in the treatment of COVID-19 virus-positive patients. Reasons for this include the anxiety of getting infected by the virus, fear of infecting loved ones, lack of personal protective equipment (PPE), and the increased number of surgeons living with some comorbid conditions. These factors have also contributed to a decreased interest in operating amongst surgeons [18]. To reduce contamination of personnel, optimize the distribution of limited surgical staff and to facilitate decision making amongst surgeons, triage algorithms, hospital pathways and designated COVID-19 staff have been recommended by the American College of Surgeons and the Surgical Society of Kenya [19]. In Morocco, dental surgeons who are at an increased risk due to involvement in aerosol-generating procedures, have been encouraged to adopt telecommunications, Pre-check triages to measure and record the temperature of staff and presenting patients, and to use particulate respirators [20].

A cross-sectional study conducted amongst paediatric surgeons in Nigeria revealed that 57 of 74 (77\%) surgeons agreed to a need for paediatric surgeons to have additional training in the management of surgical patients during epidemics; however, 6 (8\%) were neutral, while 11 (15\%) disagreed. Furthermore, 47 (64\%) were willing to attend training while 15 (20\%) were neutral and 12 (16\%) were unwilling to attend [21]. Training activities in the form of weekly seminars, journal clubs, grand rounds and clinical meetings which traditionally require physical attendance have also been negatively affected by the ongoing pandemic [20]. Surgical units are now forced to either completely discontinue these activities or adopt the use of information technology by going virtual or using internetbased tools which allow for connectivity and effective communication between multiple users simultaneously. However, this has not been particularly effective because of issues related to poor internet connectivity and the high cost of internet subscription in sub-Saharan Africa.

A survey conducted during the pandemic in Nigeria indicated a growing acceptance of video conferencing for academic activities as well as local and international conferences. $66.7 \%$ of respondents preferred online video conferencing for all academic programmes, while $44.2 \%$ of respondents already used video conferencing for weekly academic activities. Another survey conducted across 50 centres in Nigeria revealed that 29 (58\%) centres had suspended academic training during the pandemic, $13(26 \%)$ engaged "WhatsApp" chat rooms, while $3(6 \%)$ made use of Video-conferencing and 5 (10\%) still carried out their academic training through physical meetings but with social distancing [21]. Continuity of surgical training has also been affected across Africa, as various postgraduate examinations like the West African College of Surgeons and 
the South African colleges of surgeons were postponed [2224].

\section{USE OF TELEMEDICINE}

Before the COVID-19 pandemic, healthcare was primarily accessed in person for many African countries, for example, patients queuing up at physical health facilities for medical consultations, access to laboratory or medicines; compared to many high-income settings where digital health technologies have been harnessed effectively in recent decades. However, in the wake of COVID-19, the need for virtual solutions to connect physicians with their patients has increased faster than many prepared for. Examples of available telemedicine services include remote teleconsultations via voice, chat and video platforms, SMS reminders on facility appointments and mobile SMS health information dissemination and awareness for behavioural change, interactive voice recordings and artificial intelligence-powered self-triage applications [25]. According to a 2020 report by the WHO, among countries reporting health service disruptions, globally $58 \%$ of countries are now using telemedicine (advice by telephone or online means) to replace in-person consultations; in lowincome countries, this figure is $42 \%$ [26].

Telemedicine providers during the COVID-19 pandemic in Africa included private entities like Nigeria's Lafiya and Hudibia, Kenya's DactariaAfrica, Cameroon's OuiCare, Ghana's Redbird and Morocco's Dabadoc; academic institutions such as university teaching hospitals and infection disease institutes of various countries. Health facilities across Africa, both private and government-owned, that previously leveraged traditional physical consultations have adopted the teleconsultation model during the pandemic [25]. The range of services covered by the Telemedicine start-ups includes but not limited to New Consultation, follow-up visits, Management of chronic conditions, Medication prescription and delivery, Specialist consultation and referrals, as well as a host of other clinical services that can be provided remotely via secure video and audio connections through an affordable subscription plan on devices like a smartphone, tablets and virtual health kiosks $[27,28]$.

\section{IMPACT ON MANAGEMENT MODALITIES}

A cross-sectional survey conducted by European-African Hepato-Pancreato-Biliary Association (E-AHPBA), revealed that most surgical units, particularly in countries with a high number of COVID-19 cases (>100,000 cases) reported insufficient critical care capacity and reduced HPB operating sessions compared to COVID-low countries [2]. Delayed access to cancer surgery necessitated alternatives including increased neoadjuvant chemotherapy for pancreatic cancer and colorectal liver metastases, and locoregional treatments for hepatocellular carcinoma [29]. Also, Chemotherapy has become frequently used for resectable pancreatic cancer during the pandemic, particularly in COVID-high, compared to COVID-low countries [29]. Radiotherapy was not often used for resectable pancreatic cancer but used occasionally as an addition to chemotherapy in borderline resectable pancreatic cancer [29]. Given the relative chemosensitivity of colorectal liver metastases, many respondents in both COVID-low and COVID-high countries ranked chemotherapy as a widely utilized treatment modality in these cases, followed by ablation particularly in COVID-high countries [29].

The association of Gynecological Endoscopy Surgeons of Nigeria, reported that there were concerns of possible increased risk of COVID-19 transmission to healthcare workers during laparoscopic and hysteroscopic procedures [30]. This is probably due to the formation of COVID-19 contaminated aerosols during surgery, especially during intubation and from the effect of the gases used during pneumoperitoneum. Emergency surgeries are now advised to preferably be done through open surgeries than laparoscopy or hysteroscopy approach [30]. However, if or when laparoscopy or hysteroscopy is considered, health personnel in theatre must wear appropriate personal protective equipment (PPE) and all standard precautions should be observed to prevent COVID-19 infection [30].

Another survey conducted in Nigeria across 120 paediatric surgeons from 50 centres revealed that Nine (18\%) centres adopted new modalities for managing selected surgical conditions: non-operative reduction of intussusception in 1 (2\%), antibiotic management of uncomplicated acute appendicitis in 5(10\%), more conservative management of trauma and replacement of laparoscopic appendectomy with open surgery in 3(6\%) respectively [31]. In Morocco, it's currently advised to use $1.5 \%$ hydrogen peroxide or $0.2 \%$ povidone as preprocedural mouth rinse during oral surgery procedures. Extra oral dental radiographs have also been recommended as appropriate alternatives to intraoral dental radiographs to avoid stimulation of saliva secretion and coughing. Dental surgeons are currently advised to reduce aerosol production during procedures. Lifethreatening emergency cases with oral and maxillofacial compound injuries are advised to be admitted and chest Computed Tomography (CT) scan conducted if available to rule out COVID-19 infection. 


\section{RECOMMENDATIONS}

1. Quality supply chain management principles should be deployed for the procurement of Personal Protective Equipments to minimise structural deficiencies that increase the cost of healthcare and reduce access to healthcare.

2. Increased manufacturing of low-cost PPE's across African countries should be adopted to ensure adequate protection of surgeons.

3. Prioritization of emergency and life-threatening cases to non-life-threatening elective cases should be adopted across countries.

4. Aerosol generating surgeries should be minimized to reduce the spread of the virus.

5. Governments should support the development of Telemedicine infrastructure and ensure its incorporation across more surgical facilities to address the inequities in healthcare provision.

6. Creation of dedicated COVID-19 operating spaces across more surgical centres to minimize exposure of surgeons.

7. Incorporation of refresher courses on the use of PPE's and the first lines of COVID-19 management into the training of residents and hospital staff to ensure they are equipped with the right information for their protection and patient management.

8. Deployment of digital learning platforms and more simulation sessions in the training of surgeons to ensure continuity in training, in case of future pandemics.

9. Creation of well-outlined pathways and protocols for COVID-19 management.

10. More studies should be conducted to evaluate the impact of COVID-19 on Surgery in Africa and proffer solutions to the problems arising.

\section{SUMMARY}

The COVID-19 pandemic highlights the inequities in surgical systems, especially in Africa, due to poor capacity and expenditure. However, it has led to increased acceptance and incorporation of Telemedicine in surgical practice across the continent. This provides an opportunity to adopt digital technology which can address inequities in healthcare provisions and surgical training.

Author contributions: All authors were involved in concept, design, collection of data, interpretation, writing and critically revising the article. All authors approve final version of the article.

Funding: There are no people or organizations that support the study financially.

Declaration of interest: authors do not have any conflict of interests.

Data availability: Data generated or analysed during this study are available from the authors on request.
Acknowledgemens: The authors of this paper are eternally grateful to all authors that have provided sound scientific research that served as references while writing this review article.

\section{REFERENCES}

1. Sohrabi C, Alsafi Z, O'Neill N, et al. World Health Organization declares global emergency: A review of the 2019 novel coronavirus (COVID-19). Int J Surg. 2020; 76: 71-6. (doi: 10.1016/j.jjsu.2020.02.034).

2. Kapata N, Ihekweazu C, Ntoumi F, et al. Is Africa prepared for tackling the COVID-19 (SARS-CoV-2) epidemic. Lessons from past outbreaks, ongoing pan-African public health efforts, and implications for the future. Int J Infect Dis. 2020; 93: 233-6. (doi: 10.1016/j.jjid.2020.02.049).

3. Siow WT, Liew MF, Shrestha BR, Muchtar F, See KC. Managing COVID-19 in resource-limited settings: Critical care considerations. Crit Care. 2020; 24: 167. (doi: 10.1186/s13054-020-02890-x).

4. Gajewski J, Bijlmakers L, Brugha R. Global surgery Informing national strategies for scaling up surgery in sub-Saharan Africa. Int J Health Policy Manag. 2018; 7: 481-4. (doi: 10.15171/ijhpm.2018.27).

5. Martinez-Alvarez $M$, Jarde A, Usuf $E$, et al. COVID-19 pandemic in west Africa. Lancet Glob Health. 2020; 8: 631-2. (doi: 10.1016/S2214-109X(20)30123-6).

6. Hospital Beds (Per 10,000 population). WHO. Global Health Observatory data repository. 2020. Available at: https://apps.who.int/gho/data/node.main.HS07?lang=e n (Accessed: 10 May 2020).

7. M. Hitler, McCain CD, Meara JG, Hagander L, Finlayson RG. Shortage of Doctors, Shortage of Data: A Review of the Global Surgery, Obstetrics, and Anesthesia Workforce Literature. World J Surg.2014; 38(2): 269-80. (doi: 10.1007/s00268-013-2324-y).

8. Rosenthal PJ, Breman JG, Djimde AA, John CC, Kamya MR, Leke RGF, et al. COVID-19: Shining the light on Africa. Am J Trop Med Hyg. 2020; 102(6): 1145-8. (doi: 10.4269/ajtmh.20-0380).

9. Mazingi D, Navarro S, Bobel MC, Dube A, Mbanje C, Lavy C. Exploring the Impact of COVID-19 on Progress Towards Achieving Global Surgery Goals. World J Surg [Internet]. 2020; 44(8): 2451-7. (doi: 10.1007/s00268-02005627-7). 
10. Dmitri N, Omar MO, James CG, Elizabeth L, Joana FFS, Tom EFA, et al. Elective surgery cancellations due to the COVID-19 pandemic: global predictive modelling to inform surgical recovery plans. BJS. 2020; 107: 1441-49. (doi: 10.1002/bjs.11746).

11. Ademuyiwa AO, Bekele A, Berhea AB, Borgstein E, CapoChichi $\mathrm{N}$, et al. COVID-19 Preparedness Within the Surgical, Obstetric, and Anesthetic Ecosystem in SubSaharan Africa. Ann Surg. 2020; 272(1): e9-e13. (doi: 10.1097/SLA.000000000000964).

12. Mazingi D, Navarro S, Bobel MC, Dube A, Mbanje C, Lavy C. Exploring the Impact of COVID-19 on Progress Towards Achieving Global Surgery Goals. World J Surg 2020; 44: 2451-7. (doi: 10.1007/s00268-020-05627-7).

13. Salako O, Okunade K, Allsop M, Habeebu M, Toye M, Oluyede $G$, et al. Upheaval in cancer care during the COVID-19 outbreak. Ecancermedicalscience. 2020; 14: 97. (doi:10.3332/ecancer.2020.ed97)

14. Verna V, Moawia MA, Hannah S. Preparedness for COVID19 in the oncology community in Africa. Lancet Oncol 2020; 21(5): 621-2. (doi: 10.1016/S1470-2045(20)30220-5)

15. Lakhdar F, Benzagmout M. Letter: Neurosurgery at war with the COVID-19 pandemic: patient's management from an African neurosurgical center. Acta Neurochii (Wien). 2020; 162: 1787-8. (doi: 10.1007/s00701-02004406-6).

16. Ogundele IO, Alakaloko FM, Nwokoro CC, Ameh EA, Ogundele IO. Early Impact of COVID-19 Pandemic on Paediatric Surgical Practice in Nigeria: A National Survey of Paediatric Surgeons. BMJ Paediatr Open. 2020; 4(1). (doi: 10.1136/bmjpo-2020-000732)

17. Alabi OC, Okohue JE, Adewole AA, Ikechebelu JI. Association of Gynecological Endoscopy Surgeons of Nigeria (AGES) Advisory on Laparoscopic and Hysteroscopic Procedures during the COVID-19 Pandemic. Niger J Clin Pract. 2020; 23(5): 747-9. (doi: 10.4103/njcp.njcp_163_20).

18. Sunday-Nweke NA, Obasi AA. Assessing anxiety levels amongst surgeons during COVID-19 pandemic. Gen Surg: Open Access 2020; 3(1): 28-1. Available at: https://www.researchgate.net/publication/ 342261809
19. COVID-19: SSK statement on recommendations for surgical procedures and outpatient clinics. 2020; 21(1): 19. Available at: https://www.ssk.or.ke/covid-19/ (Accessed: 10 May 2020).

20. Chkoura A. Oral Surgery and COVID-19 pandemic. Int. J. Appl. Dent. Sci. 2020; 6(2): 92-5. Available at: https://www.oraljournal.com/pdf/2020/ vol6issue2/PartB/6-2-2-906.pdf (Accessed: 10 May 2020).

21. Ogundele IO, Alakaloko FM, Nwokoro CC, Ameh EA. Early Impact of COVID-19 Pandemic on Paediatric Surgical Practice in Nigeria: a National Survey of Paediatric Surgeons. medRxiv. 2020; 2020.05.24.20112326. (doi: 10.1136/bmjpo-2020-000732).

22. Shodipo OM, Abiodun AA, Toluse AM, Ogunyele OO, Wuraola FO, James JA. Impact of COVID-19 Pandemic on Surgical Practice and Training in Nigeria. IOSR J Dent Med Sci. 2020; 19(6): 32-6. (doi: 10.9790/0853-1906123236).

23. Gueye S, Mshelbwala PM. Notice of postponement of West African College of surgeons (WACS) April 2020 examinations, 2020. Available at: https://www.wacscoac.org/ (Accessed: 10 May 2020).

24. Senkubuge F. Announcement to CMSA candidates, 2020. Available at: http://cmsa.co.za/ (Accessed: 30 April 2020).

25. Kamulegeya L, Bwanika J, Musinguzi D, Bakibinga P: Continuity of Health Service Delivery During The COVID19 Pandemic: The Role of Digital Health Technologies in Uganda. PAMJ. 2020; 35(2): 43. (doi: 10.11604/pamj.supp.2020.35.2.23115).

26. WHO.int. 2020. COVID-19 Significantly Impacts Health Services for Noncommunicable Diseases. Available at: https://www.who.int/news-room/detail/01-06-2020COVID-19-significantly-impacts-health-services-fornoncommunicable-diseases (Accessed: 9 August 2020).

27. Africa S. Lafiya Telehealth is Changing the Way Medicine Works in Africa. 2020. Available at: https://www.google.com/amp/s/africanews.space/lafiy a-telehealth-is-changing-the-way-medicine-works-inafrica/amp (Accessed: 15 August 2020).

28. Asiri A, AlBishi S, AlMadani W, ElMetwally A, Househ M. The Use of Telemedicine in Surgical Care: A Systematic Review. Acta Inform Med. 2018; 26(3): 201-6. (doi: 10.5455/aim.2018.26.201-206). 
29. Balakrishnan A, Lesurtel M, Siriwardena AK, et al. Delivery of hepato-pancreato-biliary surgery during the COVID19 pandemic: An European-African Hepato-PancreatoBiliary Association (E-AHPBA) cross-sectional survey. HPB. 2020; 22(8): 1128-34. (doi: 10.1016/j.hpb.2020.05.012).
30. Alabi OC, Okohue JE, Adewole AA, Ikechebelu J. Association of gynecological endoscopy surgeons of Nigeria (AGES) advisory on laparoscopic and hysteroscopic procedures during the COVID-19 Pandemic. Niger J Clin Pract. 2020; 23: 747-9. (doi: 10.4103/njcp.njcp_163_20).

31. Ogundele IO, Alakaloko FM, Nwokoro CC, Ameh EA. Early Impact of COVID-19 Pandemic on Paediatric Surgical Practice in Nigeria: A National Survey of Paediatric Surgeons. BMJ Paediatr Open 2020; 4: e000732. (doi: 10.1136/bmjpo-2020-000732). 